\title{
La recomposition du réseau ferré allemand à la suite de la réunification
}

Recomposition of the german railway network after the reunification

Die Neustrukturierung des deutschen Eisenbahnnetzes nach der

Wiedervereinigung

\section{Etienne Auphan et Konrad Schliephake}

\section{(2) OpenEdition}

Édition électronique

URL : http://journals.openedition.org/rge/3960

DOI : 10.4000/rge.3960

ISSN : 2108-6478

Éditeur

Association des géographes de l'Est

Édition imprimée

Date de publication : 1 janvier 2001

ISSN : 0035-3213

Référence électronique

Etienne Auphan et Konrad Schliephake, « La recomposition du réseau ferré allemand à la suite de la réunification », Revue Géographique de l'Est [En ligne], vol. 41 / 1-2 | 2001, mis en ligne le 24 juillet 2013, consulté le 08 septembre 2020. URL : http://journals.openedition.org/rge/3960 ; DOI : https://doi.org/ $10.4000 /$ rge.3960

Ce document a été généré automatiquement le 8 septembre 2020.

Tous droits réservés 


\title{
La recomposition du réseau ferré allemand à la suite de la réunification
}

\author{
Recomposition of the german railway network after the reunification \\ Die Neustrukturierung des deutschen Eisenbahnnetzes nach der \\ Wiedervereinigung
}

Etienne Auphan et Konrad Schliephake

\section{Introdution}

1 Le réseau ferré allemand, qui a largement contribué à la structuration de l'espace allemand, a eu une existence singulièrement agitée, à l'image des soubresauts géopolitiques qu'a connus son fonctionnement. Mis en place au XIXe siècle sur un territoire qui s'étendait alors de la Poméranie à la Lorraine selon une structure primaire divergeant à partir de Berlin, ce réseau s'est trouvé complètement disloqué lors de la partition du pays en 1949. La Deutsche Bundesbahn, en charge d'un réseau ferré croupion amputé de son centre névralgique, s'efforcera alors tant bien que mal de l'adapter à la nouvelle configuration méridienne des flux majeurs dans le cadre de la Bundesrepublik Deutschland, alors qu'il avait été conçu pour écouler des courants principalement orientés ouest-est. A nouveau aujourd'hui, ce même réseau, appelé à jouer un rôle majeur dans la structuration du territoire du nouvel État fédéral allemand, se trouve confronté à une restructuration qui ne s'avère pas du tout un retour à la situation d'avant-guerre. 


\section{La constitution du réseau allemand dans l'espace inter-allemand}

2 A la veille de la Seconde Guerre mondiale, à la fin des années 1930, le réseau ferré allemand présente encore une très forte densité. Entre Lübeck et Bamberg, Göttingen et Halle, dans la région qui sera partagée entre deux États allemands pendant près d'un demi-siècle (fig. 1), le maillage s'avère particulièrement serré, y compris et surtout dans le massif minier du Harz et en Thuringe. On observe la remarquable primauté des liaisons principales Est-Ouest convergeant toutes vers Berlin du côté est, vers Hamburg, Hannover et Frankfurt/M. à l'ouest. Seule, la basse plaine de l'Elbe, entre Lüneburg et Stendal, apparaît un peu moins couverte par la toile d'araignée ferroviaire.

Figure 1 : La configuration du réseau ferré allemand en 1939

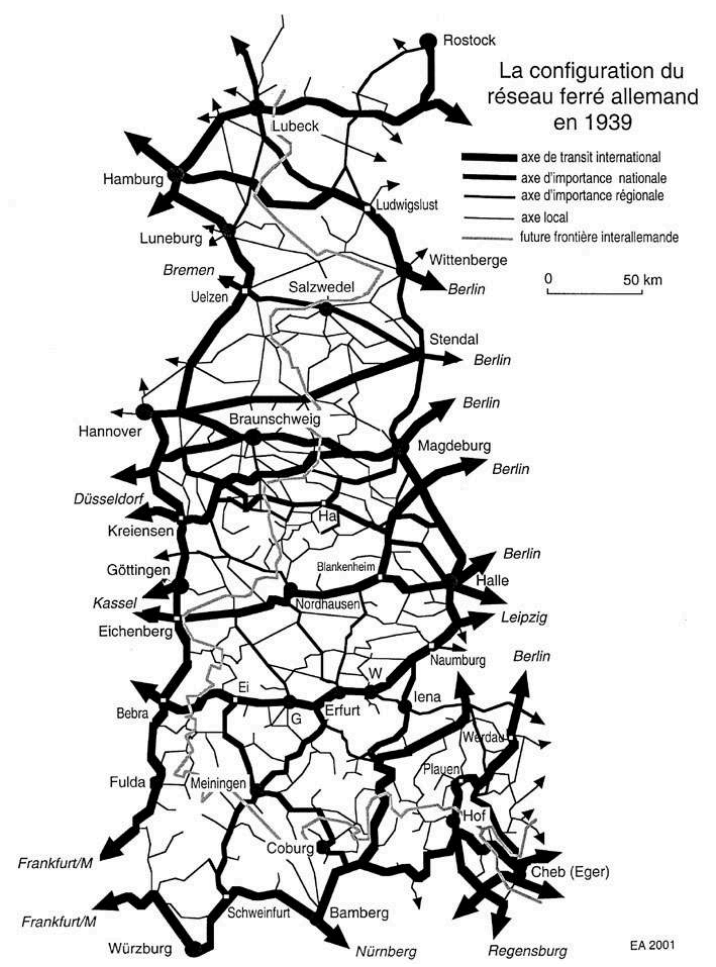

3 Ce réseau ferré est fortement hiérarchisé. On dénombre ainsi 9 axes de premier niveau, d'intérêt international :

- Hamburg-Rostock via Lübeck

- Hamburg-Berlin via Ludwigslust

- Hannover-Berlin via Stendal

- Hannover et Osnabrück-Berlin via Braunschweig et Magdeburg

- Düsseldorf-Berlin via Kreiensen et Magdeburg

- Kassel-Berlin via Eichenberg et Blankenheim et son embranchement vers Leipzig

- Frankfurt/M-Berlin via Bebra, Weimar et Naumburg, et son embranchement de Stuttgart via Würzburg

- Nürnberg-Berlin via Bamberg et Jena

- Regensburg-Berlin via Hof, Plauen et Werdau. 
Cinq autres lignes principales, mais de deuxième niveau, orientées est-ouest en Saxe et Hesse, nord-sud en Thuringe et Bavière, complètent les liaisons de premier ordre :

- Kiel-Berlin via Lübeck et Ludwigslust

- Bremen-Berlin via Uelzen et Stendal

- Hannover-Halle via Halberstadt, partiellement en tronc commun avec la ligne KreiensenMagdeburg

- Erfurt-Schweinfurt via Meiningen/Grimmenthal

- Eisenach-Bamberg via Meiningen et Coburg.

5 Vingt-quatre autres lignes secondaires (dont 3 régionales : Lübeck-Lüneburg, GoslarHeudeber et Seesen-Nordhausen) et 21 lignes locales viennent compléter le maillage très dense de cette zone devenue frontalière en 1945.

6 La Seconde Guerre mondiale fera l'effet d'un véritable séisme dans ce réseau parfaitement maillé et cohérent. La nouvelle frontière inter-allemande coupe de manière totalement désinvolte (fig. 2) l'ensemble de ce réseau en reprenant le tracé des anciennes limites politiques internes à l'Allemagne. Le fonctionnement du réseau va s'en trouver profondément modifié pour trois raisons :

- en premier lieu, les destructions de la guerre ont fait de l'Allemagne un champ de ruines, notamment en ce qui concerne les ouvrages d'art, dont plusieurs (par exemple des ponts sur l'Elbe) ne seront pas reconstruits dans la zone frontalière, engendrant ainsi la fermeture de certaines sections de ligne dans leur partie centrale (Uelzen-Ludwigslust par exemple);

- en second lieu, la mise en place du rideau de fer achèvera de «neutraliser » les sections centrales des autres lignes, interrompant ainsi le trafic de manière « provisoire » sur de nombreuses voies ferrées;

- en troisième lieu, la structure même des flux s'en trouvera totalement bouleversée, à la fois pour des raisons politiques et géographiques. 
Figure 2 : Les interruptions du réseau ferré allemand après la guerre

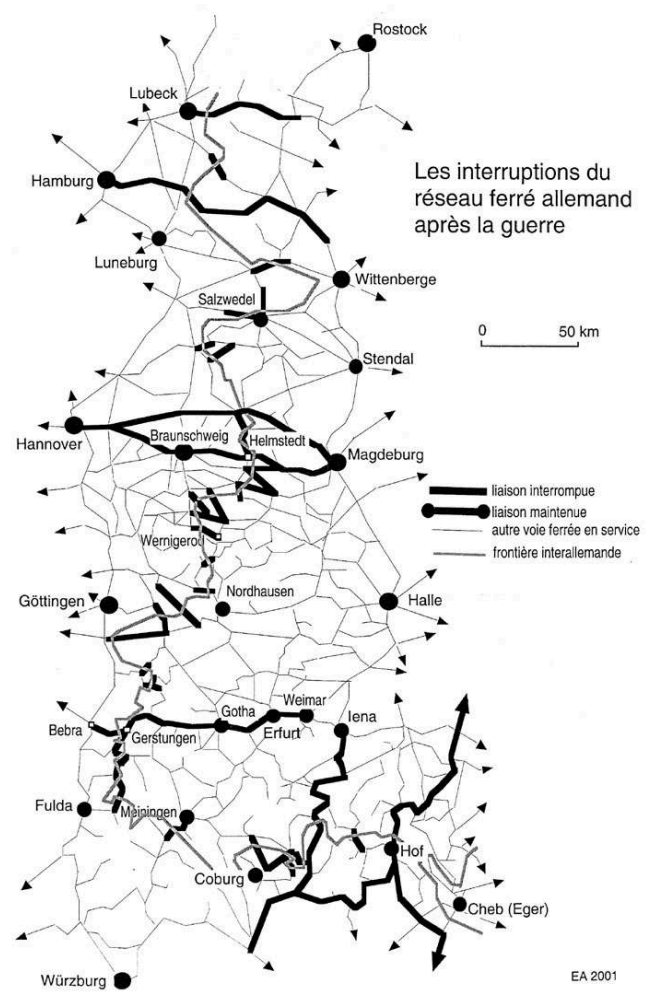

\section{La désorganisation du réseau et la restructuration des flux après la division}

7 La nouvelle frontière interallemande coupe le réseau ferré de 1939 en 43 points (fig. 2) répartis sur 37 lignes (l'une d'entre elles, Gerstungen-Kaltennordheim est recoupée en 5 endroits différents, deux autres le sont deux fois). Un quart de ces voies ferrées prennent place dans la région minière du Harz, de Helmstedt à Wernigerode, soit sur une distance de quelque $50 \mathrm{~km}$ à vol d'oiseau, une voie ferrée tous les $6 \mathrm{~km}$ en moyenne.

8 A la création des deux États allemands, en 1949, les réseaux ferrés de part et d'autre de la frontière sont réorganisés en fonction de leurs nouveaux flux internes (fig 3). Sept seulement parmi les 43 voies ferrées concernées sont maintenues en service, toutes de premier niveau. L'ensemble des autres lignes, quel que soit leur niveau, sont interrompues. Les liaisons principales [Hannover] Braunschweig-Magdeburg-[Berlin] et Kassel-Halle [Leipzig] sont même totalement supprimées. 
Figure 3 : La configuration du réseau ferré allemand pendant la division

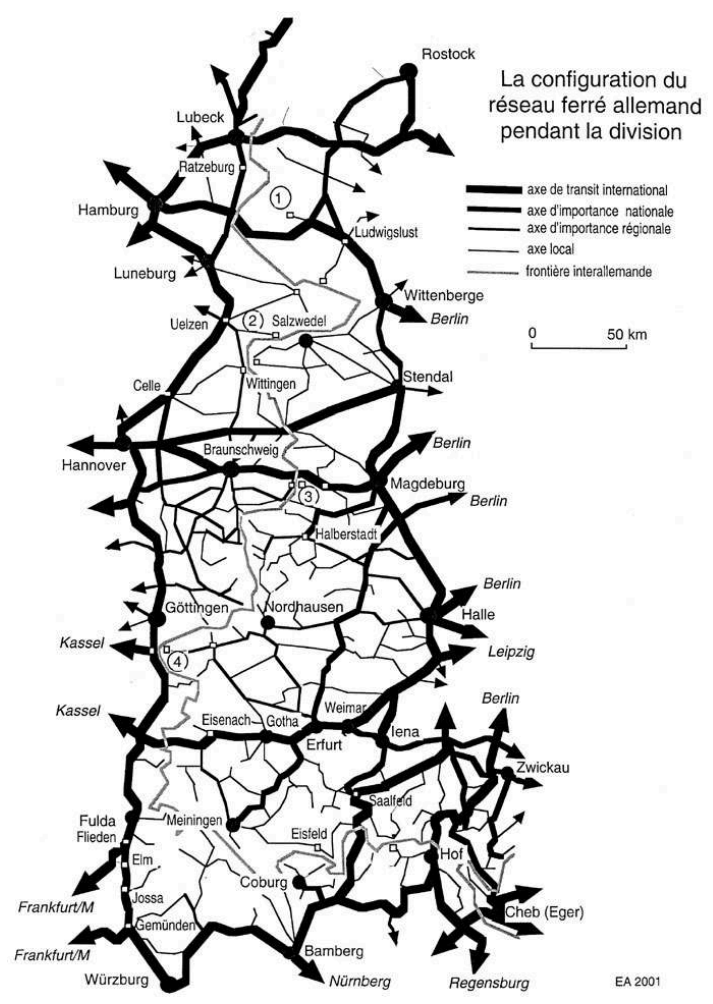

9 D'une façon générale, la coupure inter-allemande va se traduire dans la configuration du réseau ferré par la transformation de l'exploitation de ces lignes. Topologiquement, on remarque qu'aucune voie ferrée ainsi coupée ne se trouvait en impasse: toutes constituent de véritables mailles du réseau c'est-à-dire des liens se raccordant à d'autres lignes aux deux extrémités. Totalement ouvertes jusque-là en ce sens que leur desserte se prolongeait sur les lignes encadrantes avec ou sans correspondance, la rupture introduite par la nouvelle frontière aura pour effet de les transformer en antennes se faisant face de part et d'autre de celle-ci lorsqu'elles ne seront pas purement et simplement supprimées. En fait, les transformations du réseau sont de trois sortes selon le nouveau statut dévolu au lien affecté par la coupure. Le terme lien désigne ici une simple section, si brève soit-elle, considérée entre ses deux nœuds encadrants les plus proches dans l'état antérieur :

Certains liens ne seront pas remis en service à la suite des destructions de la guerre ou face aux nouvelles conditions économiques et sociales de l'après-guerre. Onze lignes sont ainsi concernées par cette fusion de mailles (fig. 4 a). Sept d'entre elles ne touchent que des maillons de liaisons locales entre des lignes de niveau supérieur. Mais les six autres constituent des sections centrales de lignes dont l'essentiel du trafic dépasse largement la section concernée. Parmi celles-ci, trois prennent place sur une ligne à caractère régional (Celle-Stendal, Uelzen-Ludwigslust, et Hof-Saalfeld), mais les trois dernières étaient intégrées dans des lignes principales de deuxième niveau écoulant un trafic de voyageurs à plus ou moins grande distance désormais totalement disparu entre des sections hiérarchiquement déclassées (fig 2):

- Hannover-Halberstadt pour sa section centrale Vienenburg - Wasserleben ;

- Würzburg-Erfurt pour la section Mellrichstadt-Ritschenhausen ;

-Eisenach-Bamberg pour la section Eisfeld-Coburg. 

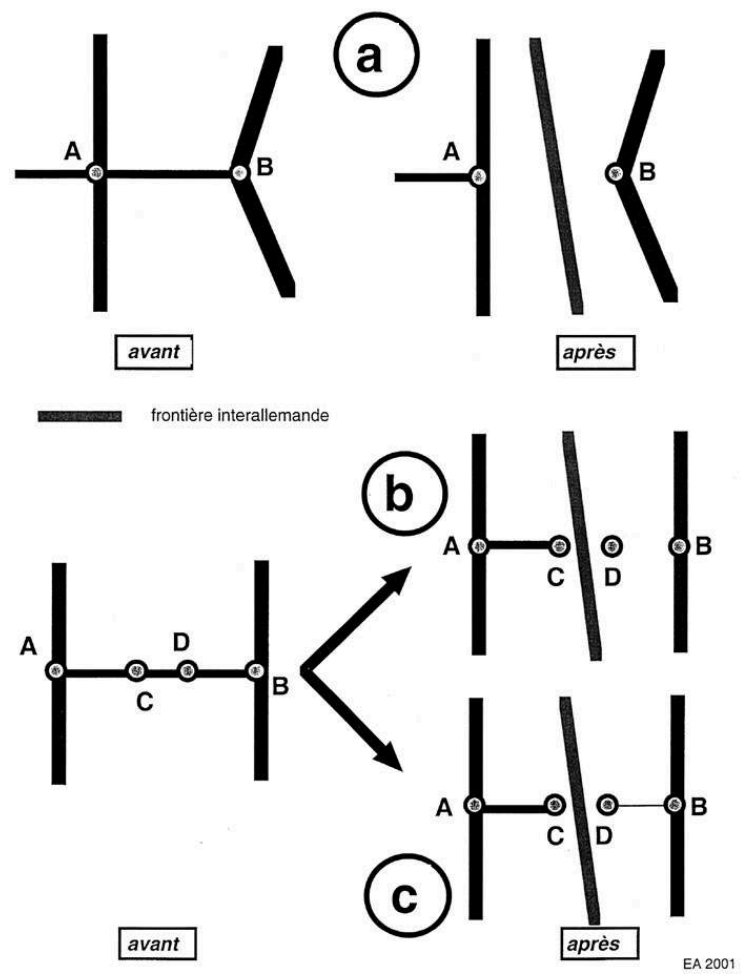
(fig. 4 b), la section restante demeurant en service sous forme d'antenne en cul-de-sac pour la desserte d'un bourg ou d'une ville à partir du réseau intérieur. A vrai dire, le maintien de cette desserte relève parfois davantage d'une mesure à caractère politique et affectif exprimant le refus d'une situation de fait, que d'une rationalité économique réelle. D'ailleurs, ces nouveaux embranchements se répartissent à parité entre les deux Allemagnes. Au nombre de 16, la plupart (une dizaine) relèvent de maillons de dernier niveau assurant des liaisons locales, mais deux d'entre eux ressortissent à des lignes régionales du Harz. Les quatre dernières sont situées sur des axes principaux qui connaissent ainsi un déclassement radical. Tel est le cas des sections suivantes :

- Ratzeburg-Hagenow Land, partie de l'ancienne ligne Lübeck-Berlin, devenue simple embranchement local de quelques kilomètres pour la desserte d'Hagenow à partir de l'axe Hamburg-Berlin (fig. $3 \mathrm{n}^{\circ} 1$ ).

-Wieren-Salzwedel, partie de l'ancienne ligne Bremen-Berlin devenue ligne secondaire à caractère local entre Uelzen et Stendal, tronçon sur lequel la section Wieren-Salzwedel n'est plus exploitée que sur sa partie ouest-allemande jusqu'à Nienbergen (fig. $3 n^{\circ} 2$ ).

- Schöningen-Eisleben, partie de l'ancienne ligne Paderborn-Magdeburg devenue ligne régionale dont la section Schöningen-Eisleben n'est plus en service que sous la forme d'un court embranchement jusqu'à Offleben (fig. $3 n^{\circ} 3$ ).

- Eichenberg-Leinefelde, partie de l'axe principal Kassel-Berlin, déclassé au rang de simple ligne régionale en Allemagne de l'est dont le tronçon Leinefelde- Eichenberg demeure en service sur le territoire est-allemand.

Sur les autres lignes traversées par la frontière interallemande, seul un tronçon central, généralement très court (quelques kilomètres) est neutralisé entre les deux gares les 
plus proches de la frontière, encadrant ainsi deux antennes exploitées en cul-de-sac (fig. 4 c). En fait, seules 3 lignes sont concernées, toutes ressortissant au niveau local. Comme dans le cas précédent, le maintien de ces dessertes traduit en premier lieu la volonté d'exprimer le refus d'une situation de fait qui explique, en dehors même du pays minier du Harz, le maintien, dans la zone frontalière, d'une densité ferroviaire supérieure à celles des deux États dans les régions voisines.

Cette réorganisation du réseau ferré de part et d'autre de la frontière inter-allemande a entraîné la restructuration des flux de voyageurs aux échelles locale, régionale et nationale. Au niveau local, le nouveau fonctionnement du réseau issu de l'interruption de trafic sur les maillons traversés par la nouvelle frontière se traduit par la suppression pure et simple du flux: le barrage de la frontière interallemande interrompt totalement les échanges locaux. Cette situation a pour effet d'accroître la contraction du réseau par le processus de «fermetures collatérales». Sous ce terme, il faut entendre la fermeture de lignes situées au voisinage immédiat du maillon dont la suppression a pour effet de ne plus leur fournir un trafic suffisant (fig. 5).

Figure 5 : Les « fermetures collatérales » (schéma)
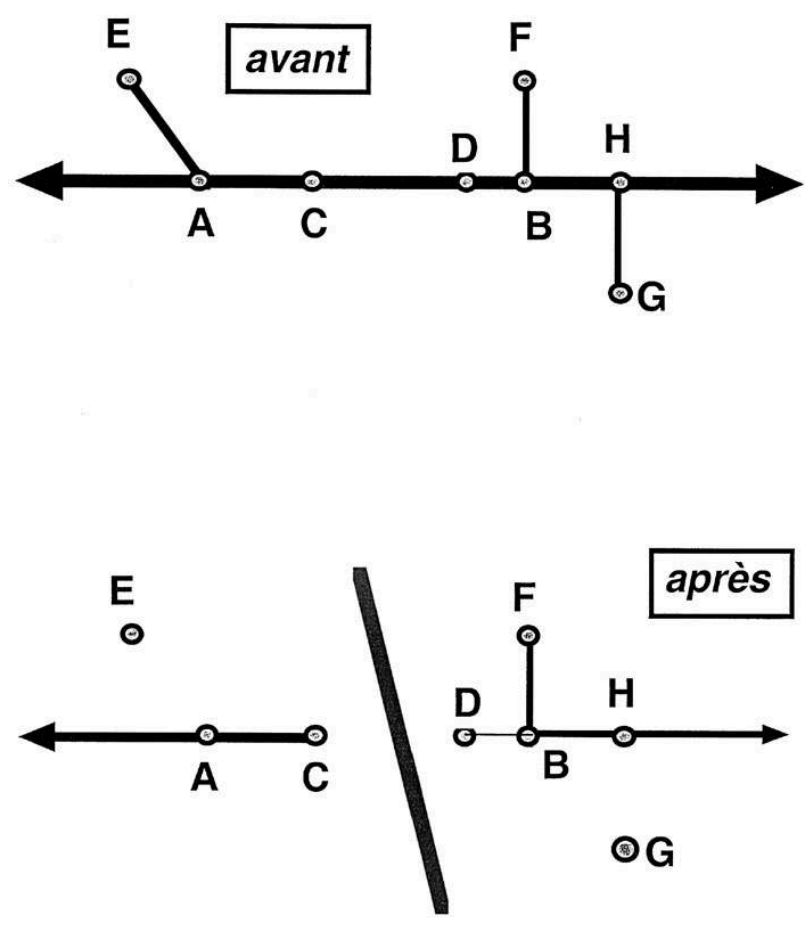

4 Au niveau régional, on assiste au phénomène de déviation de flux : le flux régional antérieurement acheminé par un itinéraire interrompu doit être reporté sur l'un des 7 « corridors » maintenus se poursuit, moyennant un détour plus ou moins important. Ainsi, le flux Brême-Stendal transitant normalement par Salzwedel doit désormais passer par Hambourg et Wittenberge; de même, la liaison Kassel-Halle empruntant traditionnellement la ligne directe par Nordhausen, est reportée via Bebra et Erfurt. Toutes ces modifications se traduisent également par le déclassement d'un grand nombre de lignes : la ligne principale de second niveau Eisfelden-Coburg est ainsi, selon 
les sections, déclassée en ligne régionale, embranchement local, ou disparaît totalement.

15 A l'échelle nationale, les flux généraux du nouvel État ouest-allemand n'ont plus grand chose de commun avec ceux du Troisième Reich. Sur ce point, c'est le fonctionnement territorial de la République Démocratique Allemande qui s'est trouvé le moins perturbé par la division du pays, l'agglomération capitale (Berlin) étant incluse (pour la partie orientale) ou enclavée (pour la partie occidentale) sur son territoire national. La structure des flux majeurs y est donc demeurée radiale, c'est-à-dire reliant la Ceinture de Berlin à la périphérie du pays: simplement, l'aire nationale desservie s'est considérablement réduite, mais la structure même du réseau n'a pas varié dans ses grandes lignes.

contraire, le fonctionnement territorial de la République Fédérale a été, dès la création du nouvel État, profondément marqué par la position - désormais extérieure du centre nerveux du réseau constitué par l'ancienne capitale politique avec laquelle d'ailleurs des liens fonctionnels tout à fait particuliers devront être maintenus à travers une continuité territoriale fictive entre le nouveau territoire national «principal » et Berlin-Ouest, isolé à plus de $200 \mathrm{~km}$ à l'intérieur de terres désormais étrangères.

En outre, alors que sur le nouveau territoire de l'Allemagne Occidentale, les flux de premier niveau de l'ancienne Allemagne étaient principalement orientés du nord-est (Berlin) vers l'ouest, le sud-ouest et le sud, afin de relier l'agglomération capitale à la Ruhr, à la confluence Rhin-Main-Neckar et à la Bavière, ceux de la nouvelle République Fédérale vont s'orienter principalement du nord au sud selon les deux axes majeurs de l'espace ouest-allemand: le "croissant fondamental » Hamburg-Ruhr-Rhin-MainNeckar-Bavière d'une part, l'axe HAFRABA Hamburg-Hannover-Frankfurt-Basel d'autre part.

Dans la région frontalière qui nous préoccupe, cette réorientation du réseau se traduira principalement par deux opérations majeures :

19 1. la réalisation de la bretelle à grand trafic Celle-Hannover (fig. 3) de manière à permettre la desserte de la métropole bas-saxonne par le trafic de grande ligne nordsud sans rebroussement.

20 2. l'aménagement en artère à grand trafic de la section Flieden-Gemunden par Jossa (fig. 3) au moyen d'une nouvelle jonction de quelques kilomètres entre Flieden et Elm, de manière à permettre l'écoulement direct du trafic à grande distance entre Hanovre et Würzburg. C'est d'ailleurs ce fonctionnement territorial interne à la RFA qui entraînera, dans le cadre du développement des échanges entre l'Europe du nord et l'Europe méditerranéenne, la saturation de l'axe HAFRABA handicapé par un tracé difficile, et justifiera la réalisation de la première Neubaustrecke ${ }^{1}$ Hannover-Würzburg, comme d'ailleurs de la seconde entre Mannheim et Stuttgart, l'une et l'autre mises en service à la veille de la réunification allemande.

\section{Le rôle du chemin de fer dans le nouveau marché de la mobilité allemande}

21 Près de 50 ans après la création du rideau de fer et la destruction des mailles d'un réseau jadis homogène, la position du chemin de fer a profondément changé, au moins dans les États ayant opté pour l'économie de marché. "Deux fois plus vite qu'en 
voiture, seulement deux fois moins vite qu'en avion ", tel était le slogan de la Deutsche Bundesbahn ${ }^{2}$ en Allemagne de l'Ouest dans les années 1980. Dans une telle perspective, les lignes secondaires n'avaient plus d'avenir, et la modernisation se concentrait sur les grands corridors, notamment sud - nord. Bien que les entités régionales, et notamment les Länder y fussent réticentes, le processus de contraction du réseau ferré s'est poursuivi sans relâche (fig. 5) : Pendant cette période, les chemins de fer ont perdu $21 \%$ de leur longueur totale, mais cette évolution est encore loin d'être terminée.

Le trafic, tant de voyageurs que de fret, demeurait assez stable, mais sa part diminuait dans un marché de la mobilité en pleine croissance (SCHLIEPHAKE 1992a, NUHN 1998). La réunification allemande en 1990 avait eu pour effet de fusionner deux entités territoriales dont les mentalités et les infrastructures étaient devenues assez différentes. A l'Ouest, le discours écologique battait son plein et la société civile, davantage que les milieux politiques, exprimait une certaine saturation devant la croissance continue du trafic issu d'une mobilité des personnes et des marchandises toujours plus développée (Schliephake 1993, et Nuhn 1998 pour le tableau général de l'Allemagne). Sur le réseau ferré, la première ligne à grande vitesse WürzburgHannover était opérationnelle depuis 1988, et l'extension de ce réseau par les autres lignes prévues (Mannheim-Stuttgart, Frankfurt-Köln et Nürnberg-München) était plus ou moins décidée.

La situation était tout autre sur le territoire de l'ex-RDA. Bien que cet État ait offert le taux de motorisation le plus élevé parmi tous les pays de l'Est (23 automobiles privées pour 100 habitants en 1989 contre 48 pour 100 à l'Ouest) les transports en commun conservaient plus ou moins leur monopole en assurant $41 \%$ des $\mathrm{v} / \mathrm{km}$ (voie ferrée et transports urbains) et $81 \%$ des $\mathrm{t} / \mathrm{km}$ (rail). Dans cette situation confortable et compte tenu de la surface relativement réduite et compacte du territoire de l'ex-RDA, la question de la concurrence et de la grande vitesse exigées par la clientèle ne se posaient guère. Certes, il existait quelques axes («magistrales») rayonnant depuis Berlin vers les capitales de provinces (Bezirke), souvent à double voie et électrifiées, mais la vitesse moyenne des Städteexpresse célébrés dans les années 1980 comme un grand événement, ne dépassait nulle part les $80 \mathrm{~km} / \mathrm{h}$.

L'unification des deux compagnies étatiques DB et DR, si différentes dans leur situation et dans leurs mentalités, était déjà une lourde tâche, mais il en était de même avec l'infrastructure, c'est-à-dire avec le réseau proprement dit. Si les projets locaux ne sont pas appelés à jouer un rôle majeur dans l'avenir pour les flux de transport, il en ira différemment avec les projets « Unité allemande ».

Tableau 1 : Développement de la mobilité personnelle en Allemagne (Est et Ouest) : parts de trafic (\%) et milliards de v/km par an de 1980 à 1999.

\begin{tabular}{|l|c|c|c|c|c|c|c|c|}
\hline & \multicolumn{2}{|c|}{1980} & \multicolumn{2}{c|}{1985} & \multicolumn{2}{c|}{1990} & 1995 & 1999 \\
\hline Mode de transport & RFA & RDA & RFA & RDA & RFA & RDA & RFA & RFA \\
\hline Chemin de fer & $7,0 \%$ & $20,4 \%$ & $7,4 \%$ & $18,4 \%$ & $6,3 \%$ & $13,5 \%$ & $8,4 \%$ & $8,0 \%$ \\
\hline Transports urbains & $13,0 \%$ & $27,8 \%$ & $12,2 \%$ & $24,3 \%$ & $9,4 \%$ & $18,9 \%$ & $8,6 \%$ & $8,3 \%$ \\
\hline Voiture individuelle & $80,0 \%$ & $51,8 \%$ & $80,4 \%$ & $57,3 \%$ & $84,3 \%$ & $67,6 \%$ & $83,0 \%$ & $83,8 \%$ \\
\hline Total en MM v/km & 588 & 108 & 580 & 122 & 705 & 133 & 895 & 916 \\
\hline
\end{tabular}

Calculé par K. Schliephake d'après Verkehr in Zahlen, 1991 ; 2000, pp. 216-217. 


\section{Les conséquences de la réunification sur la configuration du réseau ferré}

Plus de 40 ans après la division inter-allemande, la réunification des deux réseaux ferrés ne pouvait s'opérer sous la forme d'un retour à la configuration de 1939, c'est-àdire par la réouverture des 39 maillons fermés. C'est qu'entre-temps, les choses avaient bien changé, qu'il s'agisse du mode de vie, du développement économique ou du fonctionnement du système global de transport. Plus particulièrement, la contraction du réseau ferré avait été mise en œuvre, certes davantage à l'ouest qu'à l'est, et le rôle de la voie ferrée dans le système de transport et le fonctionnement des territoires étaient à redéfinir.

Au regard des liaisons à grande distance, il a été considéré que les 7 « couloirs » ouverts de part et d'autre de l'ancienne frontière suffiraient aux échanges sous réserve du réaménagement nécessaire (fig. 6) pour mettre fin à la vétusté de leur équipement en les rendant progressivement aptes à un trafic international aux normes modernes en termes de capacité et de vitesse. Dans le cadre des projets «Unité allemande » s'est inscrit par exemple l'axe Hannover-Berlin. Cette partie de l'artère intérieure magistrale Ruhr-Berlin et maillon de la grande dorsale nord-européenne Paris-Varsovie-Moscou, a fait l'objet d'une reconstruction totale. La mise en service de cette nouvelle ligne à grande vitesse (Neu- et Ausbaustrecke) Berlin-Hannover s'ajoutant aux deux précédentes (Hanovre-Würzburg et Mannheim-Stuttgart) a entraîné la capture via Frankfurt et Hannover des liaisons Berlin-Stuttgart traditionnellement acheminées par Nürnberg et Hof, ce dernier itinéraire étant désormais déclassé par voie de conséquence au rang d'axe intérieur de second niveau.

Figure 6 : Allemagne - réseau ferré en 2000, coupures et projets « Unité Allemande »

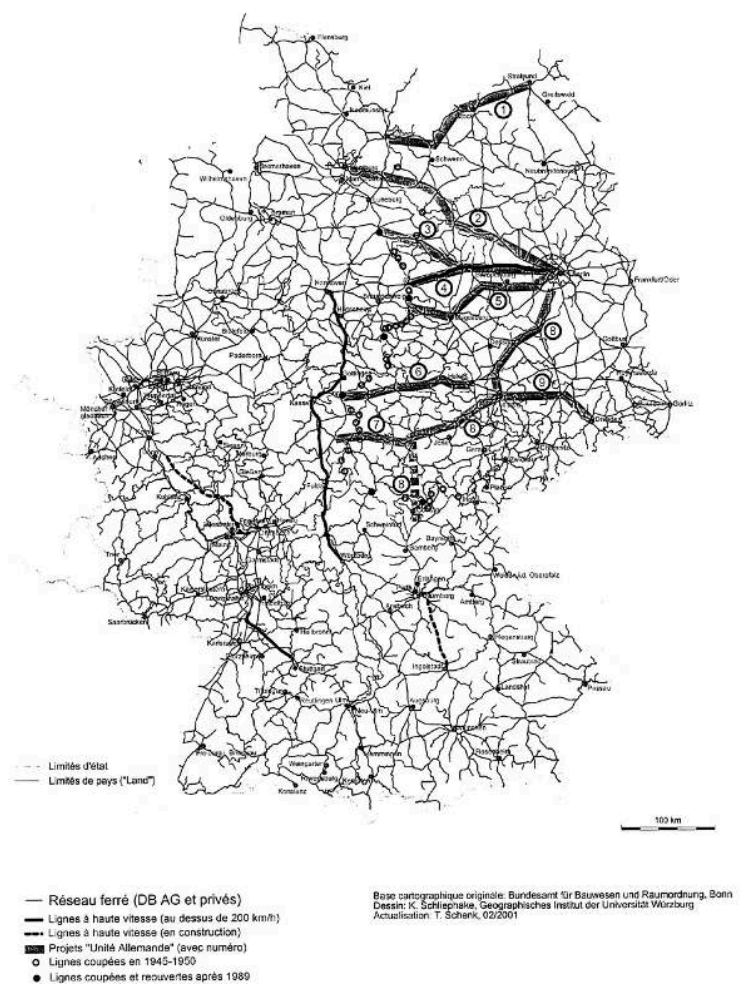


Parmi les 39 maillons victimes de la division interallemande, seuls 5 ont été, en première étape, rouverts au service ferroviaire, mais seulement à caractère régional et local :

Goslar-Halberstadt (fig.7 b) : Cette opération de réouverture sur l'ancien axe principal Hannove-Halle correspond localement aux besoins autrefois assurés par les deux maillons Vienenburg-Wasserleben et Bad Harzburg-Ilsenburg. La nouvelle jonction reprend d'abord le tracé du premier maillon à partir de Vienenburg, avant d'aboutir sur le second maillon à Ilsenburg, permettant ainsi la desserte directe de Vienenburg et de Wernigerode au détriment de Bad Harzburg à l'ouest, Wasserleben et Osterwieck à l'est desservis en antenne.

Walkenried-Nordhausen (fig. 7 c): Il s'agit là du comblement de la solution de continuité introduite entre Walkenried et Niedersachswerfen par la frontière interallemande sur la ligne régionale Hannover - Erfurt.

Eichenberg-Nordhausen (fig. 7 a): Comme dans le cas précédent, l'opération de réouverture correspond à un simple comblement d'une solution de continuité, ici encore plus courte, entre Eichenberg et Arenshausen qui peut ainsi abandonner son statut provisoire de fin de ligne tout à fait secondaire et retrouver celui de halte sur un axe régional bien desservi entre Göttingen et Kassel d'une part, Halle et Leipzig d'autre part.

31 Mellrichstadt-Meiningen. (fig. 7 d): Située en plein cœur de l'ancienne artère principale reliant la Saxe-Anhalt à la vallée du Main à travers la Thuringe, cette section a été remise en service selon le tracé de l'ancienne section neutralisée entre Mellrichstadt, devenue simple gare de passage sur l'antenne de Bad Neustadt à Fladungen, et Rentwertshausen. En outre, un raccordement entre les lignes de Mellrichstadt et d'Erfurt a été créé à l'entrée sud de Meiningen de manière à éviter un rebroussement pour les liaisons Schweinfurt-Erfurt et vice versa. (Schliephake 1992b, Schliephake \& Niedermeyer 1996).

Coburg - Sonneberg (fig. 7 e) : Il s'agit de la réouverture de la section de Neustadt (b Coburg) à Sonneberg mais d'une manière tout à fait originale. Moins qu'ailleurs, il s'agissait de recréer la ligne principale antérieure de Bamberg vers Meiningen via Coburg et Eisfeld. C'est en effet un autre maillon qui a été retenu pour la remise en service : celui de Coburg à Sonneberg, antérieurement placé sur la ligne de Meiningen à Lauscha. Cette opération avait pour objet de permettre de transférer de la voiture particulière vers le rail une partie des flux de migrations alternantes entre Sonneberg et le couloir Main/Regnitz jusqu'à l'agglomération de Nürnberg. Mais la contrepartie en a été la fermeture au trafic des voyageurs de la ligne de Meiningen à Probstzella via Sonneberg et Lauscha à partir d'Eisfeld, rendant ainsi Sonneberg terminus d'une antenne vers Coburg et la vallée du Main, c'est-à-dire selon une orientation totalement inverse à celle commandée par le fonctionnement territorial de la période des deux États allemands. 
Figure 7 : Les sections réouvertes lors de la réunification

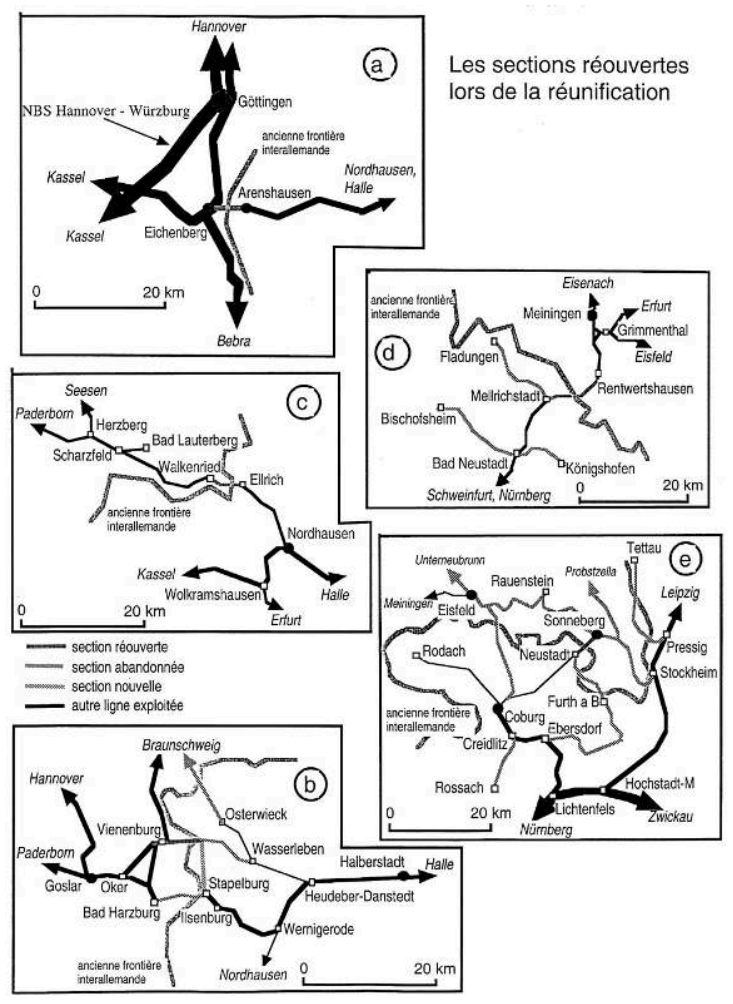

33 En fait, dans tous ces cas, il s'agit donc moins de recoudre un réseau aux mailles manquantes que de contribuer à une meilleure cohérence de territoires jusqu'alors séparés en fournissant une nouvelle accessibilité en réponse à de nouvelles demandes de mobilité régionale dans le cadre d'une politique volontariste en faveur du transport collectif.

Depuis dix ans néanmoins, la contraction du réseau ferré allemand s'est amplifiée dans l'ancienne zone frontalière comme dans le reste du pays. Ainsi, au regard de la configuration du réseau ferré, la réunification s'est traduite, par une simple adaptation légère à de nouveaux besoins, mais en aucun cas par un retour, même partiel, à la situation antérieure. Néanmoins, au-delà des modifications dans l'ancienne zone frontalière, se pose désormais plus largement le problème de la réunification des deux anciens réseaux ferrés.

\section{Les projets « Unité allemande »}

Depuis le milieu des années 1960, le Gouvernement fédéral propose des Programmes coordonnés pour le développement des infrastructures de transport fédérales ou Bundesverkehrswegpläne (BVWP). D'ailleurs, c'est le seul instrument par lequel l'État fédéral peut interférer dans la planification régionale qui, en principe, est l'affaire des Länder. Dans le cadre de ces programmes, l'État fédéral a dépensé, pour les trois périodes 1966-1975, 1975- 1985 et 1985-1989 environ 477 milliards de DM, dont environ un tiers pour le rail. Au moment de la réunification - qui a surpris les chercheurs et planificateurs des deux côtés du rideau de fer - il existait ainsi au moins un instrument institutionnel pour réaliser les nouveaux « Projets Unité allemande » : le BVWP courant 
depuis 1985 dans lequel ils furent intégrés en 1990. A cet effet, le Plan fut ainsi redéfini en 1990-1991 pour une nouvelle période à échéance de 2010/2012.

Nous n'exposerons pas ici en détail ce nouveau plan qui prévoit des investissements de 414 milliards de DM, dont - fait tout à fait exceptionnel - $47 \%$ pour le rail (Schliephake 1999 et 2000). Mais nous présenterons brièvement les 9 opérations concernant le réseau ferré (fig. 6), auxquels s'ajoutent d'ailleurs 7 projets d'autoroute et une voie fluviale (Rouyer 1994).

Projet $\mathbf{n}^{\circ} 1$ : Sur l'axe existant Lübeck-Hagenow-Rostock-Stralsund : amélioration des voies pour une vitesse maximale portée de 120 à $180 \mathrm{~km} / \mathrm{h}$ (Marquardt-Kuron 1996). Les coûts estimés à l'origine à 1,1 milliard de DM sont aujourd'hui évalués à 1, 6 milliards, dont 53 \% seront investis jusqu'en 2002, l'échéance de 1998 initialement prévue n'ayant pu être tenue.

Projet $n^{\circ} 2$ : Sur l'axe Hamburg-Büchen-Berlin : remise à double voie de l'ancienne section frontalière entre Schwarzenbek et Ludwiglust et électrification pour permettre une vitesse maximale de $160 \mathrm{~km} / \mathrm{h}$ sur la totalité de la ligne $(270 \mathrm{~km})$. Avec des investissements de 3,8 milliards de DM (somme déjà prévue en 1992), les travaux ont été terminés en 1998, autorisant une réduction de $50 \%$ de la durée du trajet BerlinHamburg, maintenant parcouru en 2 heures et 18 minutes. Cette opération a rendu caduque la construction de la ligne de «train » à sustentation magnétique Transrapid retenue dans la version 1992 du BVWP.

Projet $\mathbf{n}^{\circ} 3$ : Pour l'axe (Bremen)-Uelzen-Salzwedel-Stendal : réouverture de la section interrompue en 1945 avec remise à double voie et électrification. Cette opération, finalement réduite par le maintien de la voie unique est achevée et en service depuis 2000 sur une longueur de $107 \mathrm{~km}$ pour un coût réduit à 0,9 milliard de DM (prévision initiale : 1,85 milliard de DM).

Projet $n^{\circ} 4$ : Reconstruction et électrification de la ligne (Hannover)-Stendal-Berlin selon un nouveau tracé contournant Stendal. Longue de $260 \mathrm{~km}$, cette ligne fait partie du réseau européen à grande vitesse (200 à $250 \mathrm{~km} / \mathrm{h}$ ). Bénéficiant d'un investissement total de 5,3 milliards de DM (estimation initiale : 4,8 milliards) réalisé à $90 \%$, cette ligne est opérationnelle depuis l'automne 1998. Elle canalise aujourd'hui l'accès à la capitale allemande.

Projet $n^{\circ} 5$ : Aménagement, électrification et remise à double voie (des sections réduites à voie unique) de la ligne (Hannover)-Helmstedt-Magdeburg-Berlin, ancien "corridor" principal d'accès à Berlin au cours de la division inter-allemande. Moyennant 2,5 milliards de DM, cet axe prioritaire pour le désenclavement de Berlin (avant même le projet $n^{\circ} 4$ ) est en service depuis 1995, mais il ne permet pas des vitesses supérieures à $160 \mathrm{~km} / \mathrm{h}$.

Projet $\mathbf{n}^{\circ} 6$ : Remise en service du tronçon traversant l'ancienne frontière de 1945 de l'ancienne ligne (Kassel)-Eichenberg-Halle et modernisation sur une longueur de 170 $\mathrm{km}$ par remise à double voie et électrification. Le tronçon coupé a été remis provisoirement en service en 1990 (ci-dessus 4.) pour les seules liaisons à caractère régional (0,5 milliard de DM investi jusqu'en 1994).

Projet $\mathbf{n}^{\circ} 7$ : Réaménagement de l'axe (Kassel/Frankfurt) Bebra Erfurt. Cette partie de la liaison classique Frankfurt-Berlin (notamment après 1945) a été remise à double voie et électrifiée entre Bebra et Erfurt pour permettre une vitesse maximale de $160 \mathrm{~km} / \mathrm{h}$ en reprenant entre Bebra et Eisenach le tracé antérieur à 1945. Coupé en 2 endroits par 
l'ancienne frontière, ce dernier avait été remplacé du côté RDA en 1963 par un tronçon à voie unique reliant Gerstungen à Eisenach par Förtha en évitant la RFA. Mais cette section qui traversait une topographie très accidentée a été abandonnée en septembre 1992 après remise en service du tracé initial (Fricke \& Ritzau 1997). Pour la totalité du projet portant sur une longueur de $103 \mathrm{~km}, 87 \%$ des 2 milliards de DM d'investissements prévus ont été dépensés en 2000.

Projet $\mathbf{n}^{\circ}$ 8: Construction d'une ligne entièrement nouvelle entre (Nürnberg) Lichtenfels-Erfurt en tunnel sous la Thüringer Wald, et empruntant l'itinéraire existant vers Halle-Leipzig-Berlin. Cette ligne, d'une longueur totale de $729 \mathrm{~km}$ devait faire partie du réseau européen à grande vitesse. Mais nécessitant un investissement total de 15 milliards de DM, c'est le projet ferroviaire le plus onéreux et le plus contesté. Après l'ouverture solennelle du chantier près de Arnstadt en avril 1996 (au même moment que l'autoroute - projet $\mathrm{n}^{\circ} 16$ - Erfurt-Schwenfurt/Bamberg, (Schliephake \& Niedermeyer 1996), seules les sections existantes sont pour le moment concernées : 3 milliards de DM seront investis entre Berlin et Halle/Leipzig jusqu'en 2002. Par contre, seule la partie de la nouvelle ligne parallèle à l'autoroute (entre Erfurt et Ilmenau) a été commencée ; elle sera achevée après 2001 pour un coût de 0,6 milliard de DM. Le grand tunnel sous la montagne sera attaqué vers la fin de la période du BVWP s'il reste encore des moyens financiers à ce moment-là.

Projet $\mathbf{n}^{\circ}$ 9: Reconstruction de la voie entre Leipzig et Dresden pour permettre de hautes vitesses dans les relations vers la Pologne et la République Tchèque. Selon le projet originel, la quasi totalité de la ligne longue de $106 \mathrm{~km}$ devait bénéficier d'un nouveau tracé pour un coût de 2,7 milliards de DM. Mais en se contentant du réaménagement du tracé existant, l'investissement a été réduit à 1,9 milliards de DM dont 51 \% seront dépensés en 2002.

L'ensemble de ces projets s'effectue d'une manière régulière, mais on observe (tab. 2) que le taux de réalisation des projets routiers est plus élevé que ceux des autres modes de transport. Cela est certainement dû en premier lieu à une meilleure organisation des services fédéraux et de ceux des Länder, plus compétents pour la construction des routes. Mais d'un autre côté, certains groupes politiques sèment le doute sur l'utilité économique d'investir des fonds publics dans un chemin de fer qui a perdu son impact sur le marché de la mobilité.

Tableau 2 : Investissements totaux dans les « Projets unité allemande » par mode de transport et par année.

\begin{tabular}{|l|c|c|c|c|}
\hline \multicolumn{1}{|c|}{ Mode de transport } & $\begin{array}{c}\text { Investissements } \\
\text { totaux (MM DM) }\end{array}$ & Dont 1990-1998 & Dont 1999-2002 & Dont après 2002 \\
\hline Rail (projets 1 à 9) & 33,1 & $53 \%$ & $9 \%$ & $38 \%$ \\
Route (projets 10 à 16) & 31,3 & $37 \%$ & $35 \%$ & $28 \%$ \\
\hline Voie d'eau (projet 17) & 4,6 & $14 \%$ & $23 \%$ & $63 \%$ \\
\hline Total & 69 & $43 \%$ & $22 \%$ & $35 \%$ \\
\hline
\end{tabular}

Calculé par K. Schliephake d'après les données du Ministère fédéral du transport, du bâtiment et de l'habitat, 2000. 


\section{L'avenir du rail dans l'Allemagne réunifiée}

Les nouveaux axes à haute vitesse créés dans les deux dernières décennies du XXe siècle vont changer profondément le "paysage ferroviaire » et la position du rail sur le marché allemand du transport. L'infrastructure ferroviaire ne ressemblera plus à un réseau maillé couvrant un territoire de manière plus ou moins régulière. Par contre, les nouvelles lignes relieront exclusivement les grandes agglomérations européennes sans desservir ni la campagne, ni même les moyennes et petites villes (au-dessous de 100000 et 50000 habitants).

La figure 8 montre le changement des isochrones à partir de Berlin entre 1991 (au lendemain de la réunification) et 1999. On observe que les villes et les agglomérations majeures situées sur les lignes à grande vitesse (et notamment le projet $n^{\circ} 4$ comme en témoigne le cas de Bielefeld) ont largement profité des projets "Unité allemande" alors que pour d'autres, situées sur des relations nord-sud, la situation a peu évolué.

Figure 8 : Isochrones des voyages sur rail à partir de Berlin, 1990 et 2000

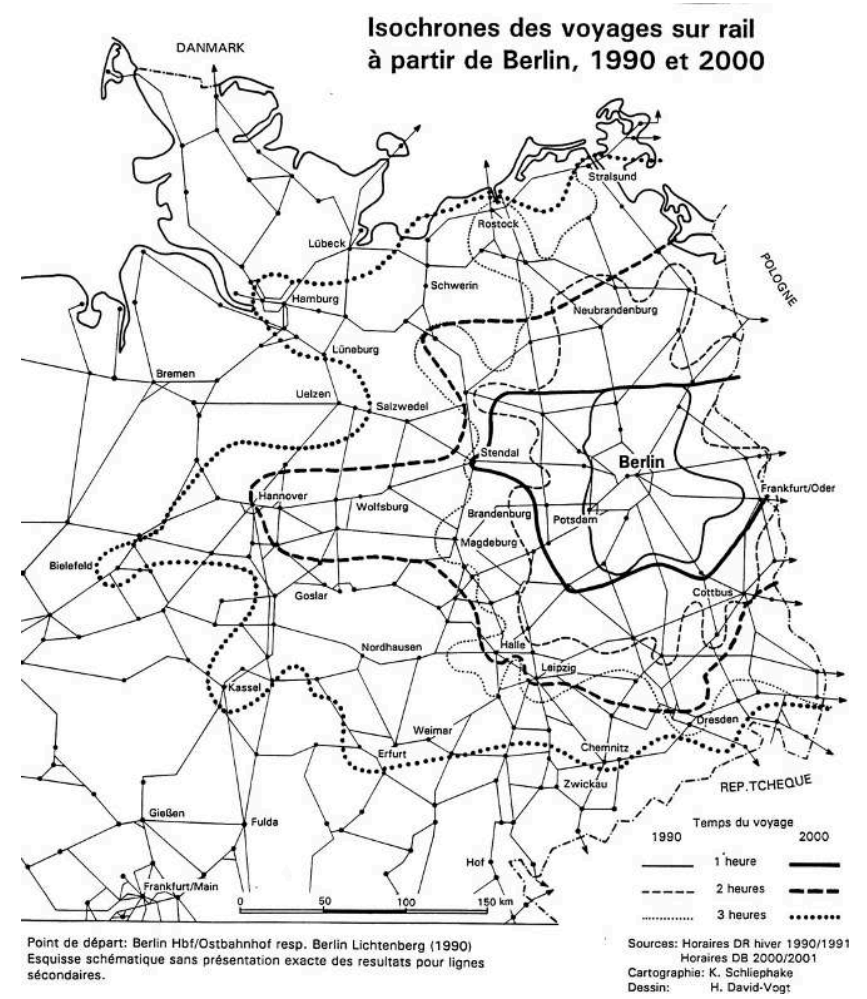

La stratégie de l'actuelle société ferroviaire allemande (la DB AG), imposée d'ailleurs par les pouvoirs publics, retient l'existence d'un réseau à grande vitesse incluant quelques lignes supplémentaires d'une longueur totale n'excédant pas 15000 à 20000 $\mathrm{km}$. L'autre moitié du réseau est confronté à l'alternative suivante :

- maintenir des trains régionaux exploités par la DB (ou ses sous-traitants) partout où les Länder en accepteront la charge financière et où l'infrastructure n'est pas trop coûteuse ;

- se défaire d'au moins $10000 \mathrm{~km}$ de lignes (sur un total de $37500 \mathrm{~km}$ ) considérés comme sans valeur commerciale pour la DB AG. Ils peuvent être vendus au plus offrant ou cédés, sur demande des États, à des entreprises de transport s'engageant à offrir des services ferroviaires réguliers. 
Ainsi, des entreprises privées, anciennes ou nouvelles, souvent appartenant à des entités régionales (ou à des sociétés multinationales, parfois d'origine française comme en Basse Saxe où opère une filiale du groupe Vivendi) ont leurs chances. Déjà elles ont agrandi leur réseau de $3000 \mathrm{~km}$ en 1990 à plus de $4000 \mathrm{~km}$ en 1999 et sont prêtes à opérer, sous réserve de subventions publiques, sur une partie des lignes secondaires délaissées par la DB AG. Mais, dans une période de "vaches maigres ", l'enthousiasme pour la réanimation des lignes secondaires en dehors des aires métropolitaines devient de plus en plus faible.

\section{Conclusion}

En raison de leur grande inertie, les réseaux ferrés s'avèrent, d'une façon générale, particulièrement peu adaptables aux modifications de flux, ce qui les rend très vulnérables aux soubresauts géopolitiques. L'exemple de l'Allemagne réunifiée montre néanmoins qu'il est au contraire possible, face à une telle situation, de transformer le réseau hérité obsolète en un outil moderne d'intégration territoriale, tant à l'échelle nationale qu'au niveau local. A condition toutefois de l'adapter au nouvelles missions du système de transport, mais aussi... d'y mettre le prix! Pour la première fois depuis des décennies, le Gouvernement allemand a fait l'effort de recréer partiellement un nouveau réseau ferré à haute qualité qui fait la fierté du public. Pour les autres parties du réseau (la majorité), la discussion sur son avenir reste ouverte. Le discours écologique si cher à certaines couches de la population allemande y contribue peut-être de manière moins convaincante que les arguments de l'évolution du coût de l'énergie (Dieters 2000). C'est la croissance de ce dernier - redouté par les nations industrialisées - qui pourrait donner un nouveau souffle à un chemin de fer de jadis qui desservait alors villes et campagnes.

\section{BIBLIOGRAPHIE}

Dieters J. (2000). - Traffic infrastructure, car mobility and public transport. In : Beiträge zur regionalen Geographie 52 : 1117-137. Leipzig.

Fricke H.J. et Ritzau H.J. (1997). — Die innerdeutsche Grenze und der Schienenverkehr. Pürgen.

Marquardt-Kuron A. (1996). - Die Autobahn A 20. - In Material zur Angewandten Geographie 26 : 275-290.

Nuhn H. (1998). - Verkehr und Kommunikation. In : E. Kulke (ed) : Wirtschaftsgeographie Deutschlands : 199-247. Gotha.

Rouyer A (1994). - La voie d'eau dans le système de transport berlinois. In : Revue géographique de l'Est 3/4 : 215-230.

Schliephake K. (1992a). - La clientèle de chemin de fer en région rurale. In : Revue d'histoire des chemins de fer, hors sér. 3, pp. 97-114. Paris. 
Schliephake K. (1992b). - Restructuration du réseau ferroviaire en Allemagne réunifiée. In : Revue géographique de l'Est 4 : 305-314.

Schliephake K. (1993). - Mobilité et transport dans l'Allemagne réunifiée. In : Recherche Transports Sécurité (Arcueil) 38/39 : 25-35.

Schliephake K. (1999). - Reunification of Germany's Transport Infrastructure. Projects and Achievements. In : J. Kitowski (ed. ) : Prace Komisji Geografii Komunikacji PTG Tom V (Dedykowane Profesorowi Teofilowi Lijewski) : 319-334. Warszawa, Rzeszow.

Schliephake K. (2000). - Verkehrsdrehscheibe Deutschland. Mobilitätszuwachs und Infrastrukturausbau im vereinigten Staat. In : Petermanns Geographische Mitteilungen 144 (5) : 58-69.

Schliephake K. et Niedermeyer M. (1996). - Infrastrukturprojekte zwischen Main und Thüringer Wald. In : Material zur Angewandten Geographie 26 : 387-408.

Verkehr in Zahlen (statistique annuelle). - Berlin (Ministère fédérale du transport, du bâtiment et de l'habitat).

\section{NOTES}

1. Neubaustrecke : ligne à grande vitesse construite ex nihilo par opposition à ausbaustrecke, ligne à (moins) grande vitesse résultant du réaménagement d'une ligne classique préexistante, de tracé favorable.

2. Entreprise publique chargée de l'exploitation du réseau ferré ouest-allemand.

\section{RÉSUMÉS}

Le réseau ferré a largement contribué à la structuration de l'espace allemand. La fin de la Seconde Guerre mondiale a engendré une profonde désorganisation de ce réseau, à la fois par les multiples coupures introduites par la mise en place de la frontière inter-allemande, mais aussi par la réorientation des flux au sein de la nouvelle République Fédérale. La réunification du pays a entrainé, à son tour, une nouvelle restructuration qui s'est opérée, d'une part par la réouverture d'urgence de 5 liaisons locales au lendemain de la réunification, d'autre part, par la mise en œuvre des opérations contenues dans le "Projet unité allemande » intégré dans le Plan fédéral révisé des infrastructures de transport. Ces opérations beaucoup plus importantes contribuent en outre à la mise en place d'un nouveau réseau ferré à grande vitesse assurant les liaisons principales à côté d'un réseau classique secondaire à l'avenir incertain. Dans le cadre des suites de la réunification, c'est donc la place et le rôle du rail dans le système global de transport qui sont ainsi remis en cause et doivent être repensés.

The development of the German railway is closely linked with the spatial structures of the prewar Germany. The Second World War destroyed and disorganised the existing network. The Iron Curtain cut more than 40 lines, and the main orientation of traffic flows changed from East-West to North-South in West Germany. The Reunification of Germany, in 1990, has altered again the structures of the railway net in two phases : reconstruction of five railway lines with rather local 
character in 1990/91; start of construction activities for the nine railway project «German Unity» within the Federal Transportation Plan 1992 to 2002. The latter projects notably add high-speed links to a network which, for 45 years, rather had a North-South orientation. In contrast to the new lines, the future of the secondary and cross- country-lines, with approx. $10.000 \mathrm{~km}$ length, is unclear. The German Railway Plc still belonging to the state- wants to reduce its activities to a trunk and high speed network. All other lines should be handed over to the Länder or regional authorities - or closed.

Der Ausbau des Eisenbahnnetzes ist eng verknüpft mit der Entwicklung der räumlichen Strukturen in Deutschland. Der zweite Weltkrieg führte zu einer Desorganisation des vorhandenen Netzes, zum einen wegen über 40 Streckentrennungen, zum anderen durch die Neuausrichtung der Verkehrsströme insbesondere in Westdeutschland. Die Wiedervereinigung 1990 hat die Strukturen des Netzes nochmals in zwei Phasen verändert : Wiederaufbau von fünf Verbindungen mit lokalem Charakter unmittelbar nach der Wiedervereinigung Beginn der Baumaßnahmen für die «Verkehrsprojekte Deutsche Einheit» innerhalb des Bundesverkehrswegeplans. Die letzteren Maßnahmen schaffen Ergänzungen zum Hochgeschwindigkeitsnetz der Eisenbahnen, das in Deutschland bislang im wesentlichen NordSüd-orientiert war. Im Gegensatz zu den neuen Strecken ist die Zukunft eines flächendeckenden Schienennetzes einschließlich der Nebenbahnen ungewiß. Die immer noch staatliche Deutsche Bahn AG will sich auf ihr Kerngeschäft zurückziehen und die Nebenstrecken den regionalen Körperschaften übergeben oder stillegen.

\section{INDEX}

Mots-clés : Allemagne, politique des transports, réseau ferré, réunification

Keywords : Germany, railway network, reunification, transportation policy

Schlüsselwörter : Ausrichtung der Verkehrsströme, Deutschland, Eisenbahnnetz, Wierdervereinigung

\section{AUTEURS}

\section{ETIENNE AUPHAN}

Université de Paris-Sorbonne (Paris IV), UFR de géographie, 191, rue Saint-Jacques, 75005 Paris.

\section{KONRAD SCHLIEPHAKE}

Geographisches Institut der Universität, Am Hubland, 97074, Würzburg. 